\title{
Effects of shocks, presented at a fixed time of day, on appetitive and general activity of rats
}

\author{
SUMIO IMADA, HIROYUKI KONDO, and HIROSHI IMADA \\ Kwansei Gakuin University, Uegahara, Nishinomiya, Japan
}

\begin{abstract}
Eating, drinking, locomotion, and rearing activities of rats, maintained under a 12:12-h dark:light cycle, were measured continuously over $24 \mathrm{~h}$ a day for 40 days in the KGbox-24. For 20 days, beginning on Day 11, the shocks were given through the grid floor of the box $1 \mathrm{~h}$ after the beginning of the dark phase (at $2100 \mathrm{~h}$ ). The daily food and water intake decreased, and there was no further gain in body weight after the introduction of shocks. The 2100 -h shocks had some associative effects: (1) Suppression of ingestion developed during the period of 2000-2100 h; (2) acceleration of general activity developed during the period of 2000-2012 h; (3) anticipatory suppression of eating developed toward the end of the light phase. The 2100-h shocks also had suppressive aftereffects upon eating and drinking, especially during the early stage of the 20day shock phase. Compensatory drinking was observed during the light phase, but no such effect was found for eating. Some findings which are not usually obtained in short-session experiments remain to be explained. References to two timing systems are made.
\end{abstract}

For an organism to survive, it has to eat food, drink water, sleep, and eliminate. For a small animal, such as the rat, to survive, it must also defend itself against predation. A well-balanced occurrence of appetitive and defensive behaviors is of utmost importance to the rat. For instance, a rat that devotes all of its activity to appetitive behavior in the presence of a threatening predator, as well as a rat that continuously demonstrates defensive behavior at the expense of eating and drinking, cannot survive. Therefore, in order to maintain its life, a rat has to learn to balance its appetitive and defensive behaviors.

Most traditional learning experiments with animals, however, have dealt with either appetitive or defensive behavior, and the interaction of the two has seldom been dealt with. A conditioned suppression situation is of interest because it involves both appetitive and defensive behaviors. But most conditioned suppression experiments concern the formation and extinction of associative processes in animals and use the suppression of appetitive behavior during CS presentation as the primary measure. In another group of studies, suppression or disturbance of appetitive baseline behavior by the introduction of aversive electric shocks has been studied for a wider time span, covering the entire session time. Among conditions that disturb an animal's appetitive behavior "chronically" (Seligman, 1968) are unpredictable and uncontrollable electric shocks (see, e.g., Imada \& Soga, 1971; Seligman, Maier, \& Solomon, 1971) or, more generally, "uncertainty" about impending aversive stimu-

This research was supported by a Grant in Aid for Scientific Researches (No. 59510074), Ministry of Education of Japan, to Hiroshi Imada. Sumio Imada is now a postdoctoral fellow, Japan Society for the Promotion of Science. Reprints may be obtained from H. Imada, Department of Psychology, Kwansei Gakuin University, Uegahara, Nishinomiya, 662 , Japan. lus conditions (Imada \& Nageishi, 1982). But even in studies that examine behavior during the entire session time, rats spend most of the time in their home cages. This may lead to the overlooking of important facts about rats' lives. For example, even if the appetitive baseline behavior of rats is suppressed completely in an operant chamber, rats can eat and drink freely and safely after they are returned to their home cages.

For those who would like to study learning as an overall process in the adaptation of the organism to an environment in which both appetitive and aversive events occur in various complex ways along a continuous flow of time, short-session experiments are unsatisfactory. Recent experiments on foraging and food economy are of interest in this connection because a free-feeding continuous session lasting $24 \mathrm{~h}$ a day is used in them (e.g., Collier, Hirsch, \& Hamlin, 1972; Collier \& Rovee-Collier, 1981; Hursh, 1980; Rashotte, O’Connell, \& Beidler, 1982). In foraging experiments, however, because of their nature, interactions of the effects of appetitive and aversive events are not of primary concern. To make all-day observation easier, Imada et al. (1983) devised a box, named KGbox24 , in which rats' activities of eating, drinking, rearing, and locomotion could be measured over $24 \mathrm{~h}$ a day for many days, and in which electric shocks could be administered through the grid floor at any time of the day.

In the first pilot experiment with this apparatus (Imada et al., 1983), the effects of shocks, given at $2100 \mathrm{~h}$ (or $1 \mathrm{~h}$ after the start of the dark phase of the 12:12-h dark:light cycle), upon various activities of a rat were investigated for 10 days. The results of that experiment can be briefly summarized as follows: (1) A typical nightacti' 2 pattern was observed for all behavioral measures in both shock and nonshock phases. (2) With the introduction of the 2100-h shocks, the daily total amount of food and water intake decreased and did not recover for 10 
days. (3) Eating and drinking during the period of 2000 $2100 \mathrm{~h}$ became completely suppressed. (4) Rearing and locomotive activities during the period of $2000-2100 \mathrm{~h}$ gradually decreased for the first 8 days and then showed an abrupt increase on the last 2 days. (5) Anticipatory suppression of eating toward the end of the light phase developed gradually. (6) Suppressive aftereffects of the $2100-\mathrm{h}$ shocks on eating and drinking became greater as the days progressed.

Although these findings show interesting local (2000$2100 \mathrm{~h}$ ) and overall effects of the 2100 -h shocks, they have limited generality because the study used a single subject. The primary purpose of the present experiment, then, was to ascertain the generality of the previous findings by using more rats. The secondary purpose was to determine if rats would develop any new strategy for better survival when the shock phase was extended from 10 to 20 days. Would rats, during a prolonged shock phase, show any sign of making up the expected weight loss, caused by suppression of eating and drinking due to the shocks given at a favored meal-time? The effects of shock on the body weight of rats were then assessed with reference to the normal body-weight curve of nonshocked rats. The purpose of the present study, stated more specifically, was to study the effects of 2100 -h shocks upon the eating, drinking, and general activities of rats over $24 \mathrm{~h}$ a day for 20 days. For purposes of comparison, this treatment was preceded by and followed by nonshock phases of 10 days each.

\section{METHOD}

\section{Subjects}

Twelve 10-week-old experimentally niave male albino rats of the Fischer strain (F344/DuCrj) were obtained from Charles River Japan Inc. They were divided into two groups of six rats each, matched in body weight. One group of six rats served as the home-cage control and was placed under a continuous ad-lib food and water condition in their home cages in the colony. They were weighed daily at the same time the experimental rats were weighed. The 12:12 dark:light cycle was the same in colony and in the experimental box described below.

Since it took a minimum of 54 days to run the experiment per se, and two rats were run at a time, the rats were purchased on three different occasions.

\section{Apparatus}

Two units of the KGbox-24 (Imada et al., 1983) were used, one for each rat. The floor plan of the apparatus is shown in Figure 1. Measures of eating, drinking, rearing, and locomotion could be recorded for $24 \mathrm{~h}$ a day over days. The inside dimensions of the box were $36 \times 18 \times 20 \mathrm{~cm}$ high. The front and rear walls and the top of the box were made of tansparent Plexiglas $(5 \mathrm{~mm}$ thick, and the left and right walls were made of stainless steel plates. The floor of the box consisted of stainless steel grid bars $(5 \mathrm{~cm}$ in diameter) spaced $17 \mathrm{~mm}$ center to center. As shown in Figure 1, a drinking tube $(8 \mathrm{~mm}$ in diameter) was located behind the right wall, and a food trough was attached to the left wall. The rat could gain access to the drinking tube through a hole (12 $\mathrm{mm}$ in diameter) approximately $60 \mathrm{~mm}$ from the grid-floor surface and $50 \mathrm{~mm}$ from the front wall (as measured at the center of the hole). The tube was round-tipped and contained a valve-loaded nozzle (Model TV-25, SE Co.). A food trough, diagonally opposite to the drinking tube, had a $4 \times 6 \mathrm{~cm}$ opening. A head-poking movement through the

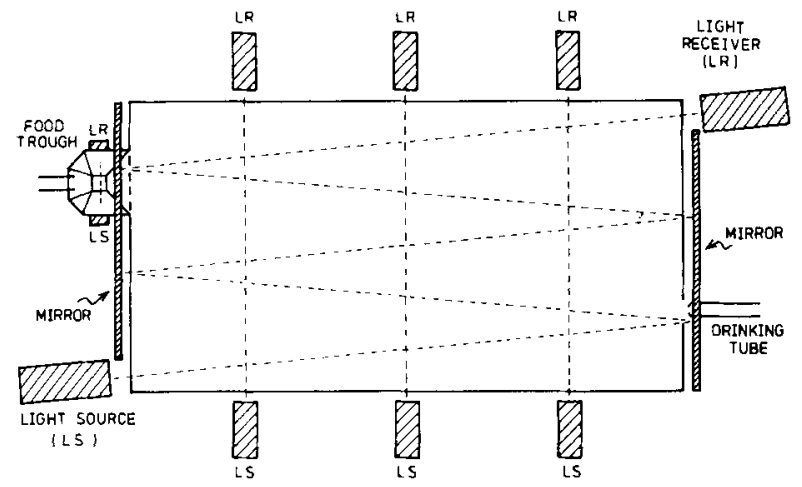

Figure 1. Floor plan of the apparatus, KGbox-24.

opening into the food trough activated a peliet dispenser by interrupting a photobeam $1 \mathrm{~cm}$ inside of the food trough as measured from the surface of the wall. The food trough was tapered to a base, $1 \times 1 \mathrm{~cm}$ square, which formed the bottom of the trough. A thin photobeam (generated by OPE-S3, OMRON) was located just above the base, and when a pellet or pellets of food interrupted this beam, a head-poking movement was nonfunctional in releasing a pellet. The pellets were a hard, 95-mg variety purchased from O'Hara \& Co., Ltd., Tokyo.

Locomotive activities were measured by three sets of photoswitches (OPE-S100, OMRON), placed $105 \mathrm{~mm}$ apart at approximately $35 \mathrm{~mm}$ from the grid-floor surface. Since the beams could be cut by movements other than locomotion (sometimes even by breathing), in cases when interruptions of the same beam occurred more than twice without being intervened by the cutting of any other beams, only the first interruption was counted. Rearing activities of greater than $15 \mathrm{~cm}$ were detected by using a method originally used by Shishimi and Imada (1977).

During the light phase of the experiment, two minilamps illuminated the box. They were located $35 \mathrm{~cm}$ above the grid-floor surface and shed 40 -lx light onto the grid-floor surface through a transparent ceiling.

The entire box was installed in a sound-attenuating box $(70 \times$ $44 \times 49 \mathrm{~cm}$ high, i.d.). A pellet dispenser and a graduated cylinder were placed outside the sound-attenuating box. Thus, the experimenter could fill the feeder with pellets and the cylinder with water at any time, throughout the experiment, without interrupting a rat's behavior.

The electric shock was a constant-voltage scrambled grid shock and was delivered through a $250-\mathrm{k} \Omega$ current-limiting resistor in series with the rat. Its intensity was $240 \mathrm{~V}$ ac, approximately $0.69 \mathrm{~mA}$, assuming the resistance of a rat to be $100 \mathrm{k \Omega}$. All the recording and the control of stimuli were accomplished using a programmable controller (SYS-P0R10, OMRON) and a microcomputer (M100OACE IV, SORD).

The temperature inside the sound-attenuating box was kept at approximately $25^{\circ} \mathrm{C}$, and the box was ventilated throughout the experiment by a fan, which produced a masking noise of $73 \mathrm{~dB}(\mathrm{C})$.

\section{Procedure}

The light phase started at $0800 \mathrm{~h}$, and the dark phase at $2000 \mathrm{~h}$, a 12:12-h light:dark cycle on which the rat had been maintained in the colony for 2 weeks preceding the experiment. After 10 days of adaptation to this light:dark cycle in the KGbox-24, the shocks were given for 20 days followed by a recovery session of 10 days. At $2100 \mathrm{~h}$ ( $1 \mathrm{~h}$ after the beginning of the dark phase), eight 3-sec scrambled grid shocks were delivered with an intershock (offsetonset) interval of $12 \mathrm{sec}$. Drinking, eating, locomotion, and rearing of the rat were recorded automatically at 3-min intervals. At 1345 h every day, the entire system was stopped for $15 \mathrm{~min}$, during which all the data stored in the memory of the computer were 
transferred to a floppy disk, the amount of water consumed during the previous $233 / 4 \mathrm{~h}$ was measured, the graduated cylinder was filled with water, and the dispenser filled with pellets.

In the previous experiment (Imada et al., 1983), rats were tested without interruption throughout the experiment. In the present experiment, however, immediately after $1345 \mathrm{~h}$ every day, the rat was taken out of the box once and weighed. During this time, the inside of the box and the mirrors used to detect the rearing responses were cleaned. Then the rat was returned to the box. Measurements resumed at $1400 \mathrm{~h}$. The rats were weighed daily because that was considered to be an important measure of a rat's survival processes.

\begin{abstract}
Measures
The measures used were the following: Eating-The number of times the pellet dispenser operated. Drinking-The amount of water intake per lick $(\mathrm{P})$ was calculated from the total amount of water intake $(A)$ and the total number of tongue licks $(T), P=A / T$. Then any local amount of water intake in milliliters was calculated by multiplying the local number of licks by P. Rearing - The number of times a rat cut the photobeam at a $15-\mathrm{cm}$ height. LocomotionIn the present apparatus, a rat had to move from one end of the box to the other when it switched from eating to drinking, or vice versa. It was, therefore, inappropriate simply to count the total number of interruptions of the three lower beams and to make it a measure of "general" locomotive activity, inasmuch as such a measure would obviously be confounded by locomotions associated with consummatory behaviors. The corrected score was obtained as follows: Assuming that a rat had to interrupt at least two beams when it switched from eating to drinking, or vice versa, two beam cuttings were subtracted from the locomotion scores obtained in any 3-min unit in which a switching in consummatory behavior had occurred and/or from those of the preceding unit or units. The total number of beam cuttings thus corrected was a measure of general locomotion.
\end{abstract}

\section{RESULTS AND DISCUSSION}

\section{Body Weight and Total Daily Amount of Food and Water Intake}

The top panel of Figure 2 shows the changes in body weights of both the home-cage control group and the experimental group in blocks of 2 days; the period covered includes the 4 days immediately preceding placement of the experimental rats into the experimental box. For the first 10 days (Blocks 1 through 5), the means of the body weights of both groups increased gradually, showing the normal growth curves. After the beginning of the shock phase, however, although the control rats continued to gain weight, no further noticeable growth occurred in the experimental rats. The results of the $2 \times 6$ ANOVA conducted on this measure for the shock phase and the last 2 days of the nonshock phase, combining two adjoining blocks together, showed the main effects of both blocks and groups to be significant $[\mathrm{F}(5.55)=43.88, \mathrm{p}<.001$; $\mathrm{F}(1,11)=10.01, \mathrm{p}<.01$, respectively]. The interaction effect of these two terms was also significant $[F(5,55)$ $=29.74, \mathrm{p}<.001]$.

It is obvious from Figure 2 that the administration of shocks at $2100 \mathrm{~h}$ had a substantial detrimiental effect on the growth of the rats. The mean body weight of the experimental group was $91.0 \%$ of that of the control group in the last block of the shock phase. After omission of the shocks, the experimental rats immediately resumed a normal growth rate, as indicated by the curve for the

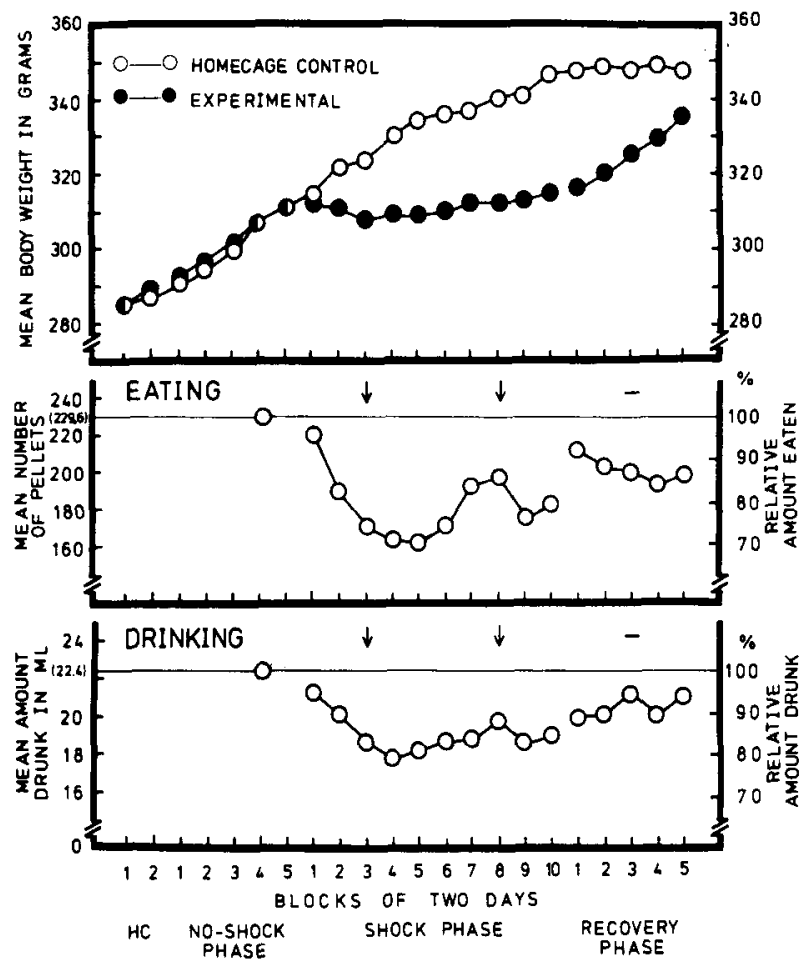

Figure 2. The upper panel shows changes in body weight for the home-cage control and experimental groups. The lower two panels show changes in the total daily amount of food and water intake. The horizontal line shows the baseline. The arrows indicate the 10day blocks that differed significantly from the respective baselines. $\mathrm{HC}=$ home cage.

recovery phase: The parallel displacement of this part of the curve along the abscissa toward the left, to the point at which the curve meets that of the control group, shows that the slopes of the curves are nearly identical.

Closely related to the body-weight changes are, of course, the total daily amount of food and water intakes, which are shown in the lower two panels of Figure 2. Since no record other than that of body weight was taken for the control group, all the descriptions below are for the experimental rats. The means of the amounts of food and water intake on the last 4 days of the nonshock phase were regarded as forming baselines and are shown by horizontal lines in Figure 2. For the purpose of statistical tests of significance, the data were combined for each measure for Days 1-10 and 11-20 of the shock phase and for 10 days of the recovery phase. The 10-day blocks that differed significantly $(p<.05)$ from the respective baselines are shown in each panel by the upward (significant increase) and downward (significant decrease) arrows. This same indication is used in all the following graphs.

The following summarizes the results: (1) During the shock phase, the total daily amount of food and water intake decreased significantly from the baseline amount. (2) During the recovery phase, the total daily amount of both food and water intake recovered somewhat, but the recovery was not complete, especially in terms of food intake. 
It is of interest to note that, although the body-weight curve obtained during the recovery phase was a continuation of the curve obtained during the nonshock phase, the daily food intake did not completely return to the baseline. This seems to suggest that experimental rats, after having experienced shocks for 20 days, developed some effective internal process for good nutrient utilization.

\section{Results for 2000-2100 Hours}

The procedure for administering electric shocks at a fixed time of day is, in principle, that of aversive temporal conditioning, and the overall daily behaviors of the rats could be analyzed with reference to this temporal regularity of the schedule. The present procedure, however, can better be considered as ordinary classical aversive conditioning, in which the putative conditioned stimulus (CS) was the termination of light, the interstimulus interval was $1 \mathrm{~h}$, and the intertrial (CS-onset-onset) interval $24 \mathrm{~h}$. The results for the period of 2000-2100 h were then analyzed separately from those obtained for the rest of the day.

The left panel of Figure 3 shows the mean amount of water drunk and the mean number of pellets eaten during the first hour of the dark phase $(2000-2100 \mathrm{~h})$ in blocks of 2 days. The first block is for the 4 days immediately preceding the shock phase. The right panel of Figure 3 shows the data for locomotion and rearing summarized in the same way as in the left panel. To facilitate comparisons between measures, the means for all four measures for Block 1 were adjusted to the same point along the ordinate, which is shown by the horizontal line in Figure 3. This forms the baseline value for each measure, the actual values of which are shown along each ordinate in parentheses. Comparisons of the baseline data with the combined data for 10-day blocks were attempted and are shown in the same way as in Figure 2. Although the drinking level for the first 10-day block of the shock phase did not differ significantly from the baseline, this was due to the results of one rat, which happened to have a very low baseline and a very high drinking rate on Day 1 . All the drinking scores for the remaining 9 days of the first 10day block were virtually zero.

Systematic developments of suppression are clearly evidenced for eating and drinking. The fact that the amount of suppression of consummatory behaviors was much greater in the hour prior to shock than it was for the entire day (see lower two panels of Figure 2) suggests that the former effect was mainly due to conditioning. The changes in locomotion and rearing were more complicated and less systematic than those for consummatory behaviors, and it is less certain that they were due to conditioning. The activity data were then analyzed in more detail, and the results are given in Figure 4 in 5-day blocks. Figure 4 shows the changes in the amount of locomotion (left panel) and of rearing (right panel) during 72-min periods, beginning at $1948 \mathrm{~h}$, in blocks of $12 \mathrm{~min}$. The data for the first day of the shock phase and the first day of the recovery phase were included in the baseline and shock-phase data, respectively. Thus, the last block of the recovery phase consists of 4 days. Systematic changes in both locomotion and rearing occurred mostly during the first 12 min immediately after the beginning of the dark phase. During the shock phase, the amount of both locomotion and rearing during the period of 2000-2012 h gradually increased; they rapidly decreased during the nonshock phase. The effects of blocks of days were significant for both locomotion $[\mathrm{F}(6,30)=5.80, \mathrm{p}<.001]$ and rearing $[F(6,30)=2.61, p<.05]$ for the period of 2000-2012 h. For locomotion, the effect of blocks of days was significant also for the period of 2012-2024 $\mathrm{h}[\mathrm{F}(6,30)$ $=2.44, p<.05]$. Figure 4 , then, clearly shows that the effects of conditioning and extinction upon general activities were most remarkable during the first $12 \mathrm{~min}$ of the "CS."

Since the present experiment differed from standard conditioning experiments in many ways, the following remarks are in order: (1) Suppression of consummatory behaviors appeared only after two "trials," which would

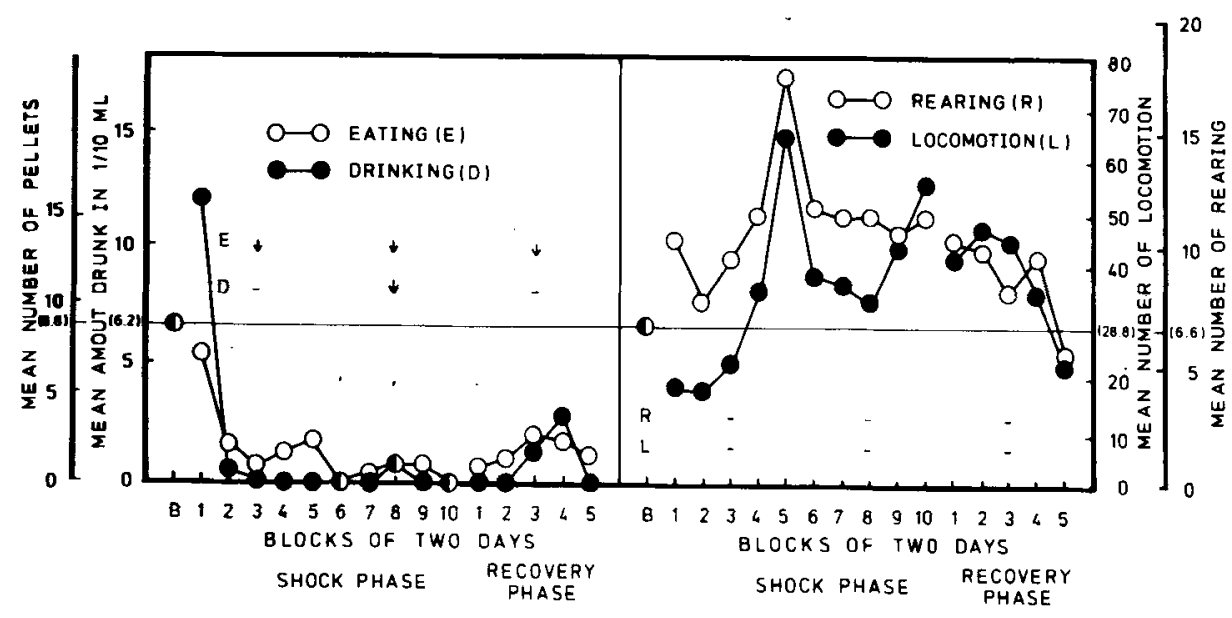

Figure 3. Changes in the amount of food and water intake (left panel) and in locomotion and rearing (right panel) in blocks of 2 days during the period of $2000-2100 \mathrm{~h}$. Block B (base) is a 4-day block. 


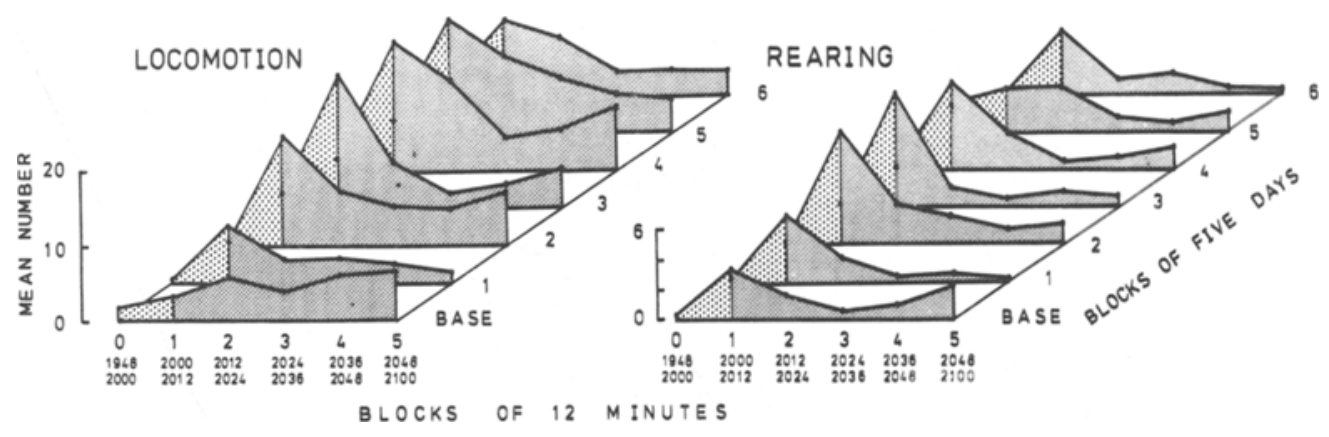

Figure 4. Changes in the amount of locomotion and rearing in blocks of $12 \mathrm{~min}$ during $1948-2100 \mathrm{~h}$ over a period of 5 days. See text for details.

make one suspect the appropriateness of a conditioning interpretation of the present results. It should, however, be noted that the present results were obtained under the condition in which both food and water were given ad lib (cf. Moran, 1975). (2) Although consummatory behaviors were suppressed, general activity increased with repeated shocks. This contradicts the generally held view that conditioned suppression of appetitive behavior is due to freezing, one of the rat's SSDRs, or species-specific defense reactions (e.g., Bolles \& Fanselow, 1980; Bouton \& Bolles, 1980). The finding of conditioned acceleration of general activities also contradicts the finding of conditioned suppression of exploration observed in shortsession experiments (e.g., Davitz, 1955). (3) Why conditioned acceleration was observed for both locomotion and rearing during the first $12 \mathrm{~min}$ of the "CS" and not in the following period is not clear. (4) The fact that the beginning of the dark phase was the "CS" onset in the present experiment may place limitations on the present findings as representing general phenomena for classical aversive conditioning with the CS-US interval of $1 \mathrm{~h}$. It should also be remembered that the light offset, which was shown to be an effective CS in the present experiment, is generally considered to be a fairly ineffective CS (e.g., Jacob \& LoLordo, 1977, 1980).

The present experiment can also be discussed with reference to the literature on circadian rhythms. For example, Terman, Gibbon, Fairhurst, and Waring (1984) ran rats on a 24-h basis in the absence of a day-night illumination cycle, with the food available for $4 \mathrm{~h}$ at a fixed time of day. Even under the uncued condition, the rats showed premeal anticipatory response accelerations, thus suggesting the presence of a circadian timing system. Terman et al. also found evidence of the presence of a shortinterval timing system under the condition with a premeal cue. Thus, it seems reasonable to assume that both of the above timing processes worked concurrently in the rats of the present experiment and determined the response patterns during the period of $2000-2100 \mathrm{~h}$.

\section{Results for Hours 2113-0800, 0800-1345, and $1400-2000$}

Figure 5 shows the results for the periods $2113-0800 \mathrm{~h}$ (dark phase), 0800-1345 h (light phase 1) and 1400-
$2000 \mathrm{~h}$ (light phase 2) for four measures separately. The data for the first 10 min immediately after the shock administration were omitted because they were affected by the unconditional aftereffects of shocks. The light-phase data obtained before and after the subject was weighed were treated separately and are drawn by two curves.

The following is a summary of the results and some related discussion: (1) The night-active pattern was observed for all measures. (2) Eating behavior was suppressed during both dark and light phases. (3) Drinking behavior was suppressed during the dark phase, but there was compensatory drinking during the light phase. Drinking data combined for the two light periods (0800-1345 h and 1400-2000 h) differed significantly from baseline even during the first 10-day block of the shock phase. (4) Thus, eating and drinking were affected differentially by the 2100-h shocks. This fact is of interest in light of Fitzsimons and Le Magnen (1969) finding that at least $70 \%$ of the drinking occurs in close association with eating. (5) Both locomotion and rearing for the period 2113$0800 \mathrm{~h}$ increased, especially during the early part of the 20-day shock phase. (6) Locomotion and rearing during the light phase were little affected by the administration of 2100-h shocks: None of the 10-day-block data differed significantly from the respective baselines.

To examine the effects of the 2100 -h shocks on the patterns of consummatory behaviors over a wide range of time, midpoint-value analyses of the data were attempted, as in Imada et al. (1983); the results are summarized in Figure 6. In this analysis, the clock time in which half of the total activity took place during a given period of time was calculated, as in the calculation of median values. For example, the means of the midpoints for eating and drinking during the period $2113-0800 \mathrm{~h}$ for the 4 days immediately preceding the shock phase were 0236 and $0222 \mathrm{~h}$, respectively. With the introduction of the 2100-h shocks, the means of the midpoints during the first 2 days of the shock phase were displaced to 0305 and $0328 \mathrm{~h}$ for eating and drinking, respectively. These findings are summarized in the right half of Figure 6, which shows the aftereffects of the 2100-h shocks upon consummatory behaviors during the dark phase.

The following is a summary of the results and some discussion pertaining to Figure 6. (1) The effects of the 


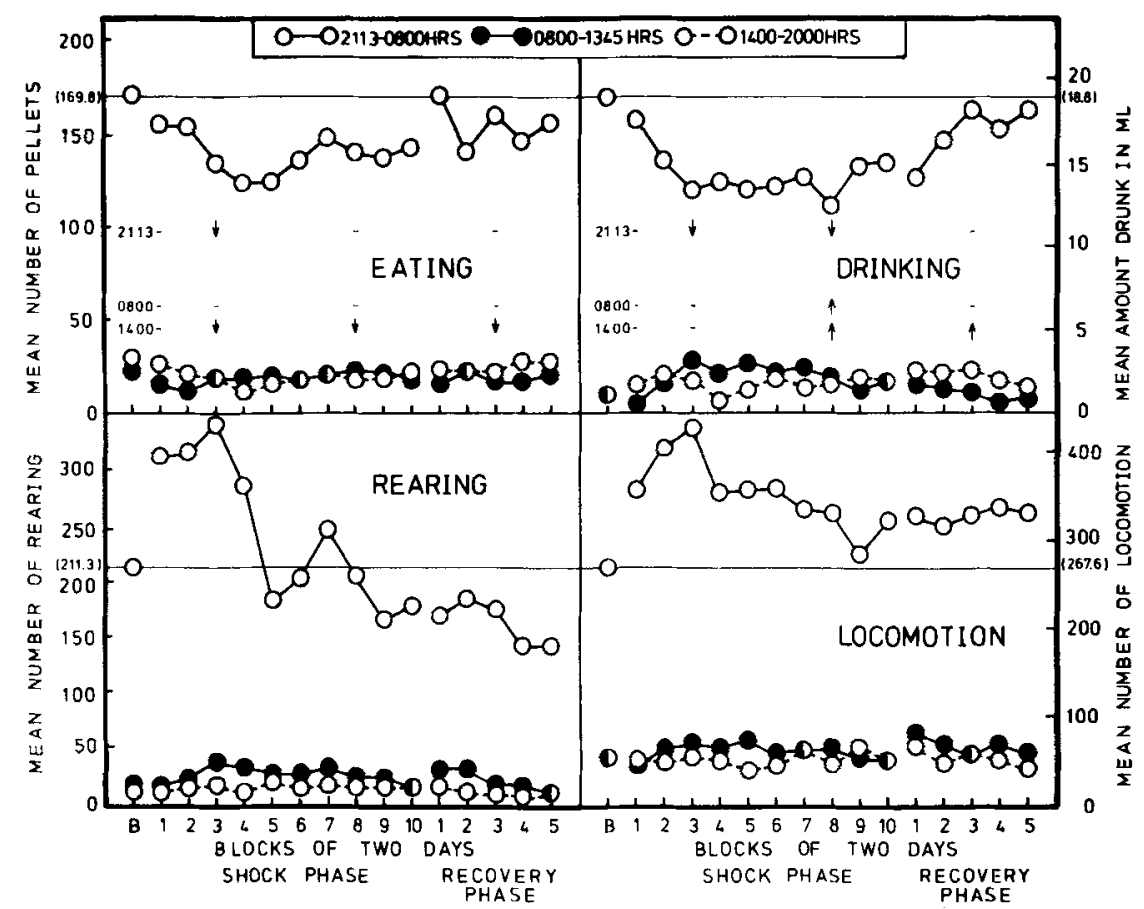

Figure 5. Changes in the amount of food and water intake in blocks of 2 days for the periods of 2113-0800 h, 0800-1345 h, and 1400-2000 h. Block B (base) is a 4-day block.

2100-h shocks upon consummatory behaviors were greatest in the second block of the shock phase; thereafter, they gradually decreased. (2) The effects of the shocks upon drinking were greater and longer lasting than those upon eating. (3) The midpoint values for eating during the period of $1400-2000 \mathrm{~h}$ were clearly displaced to

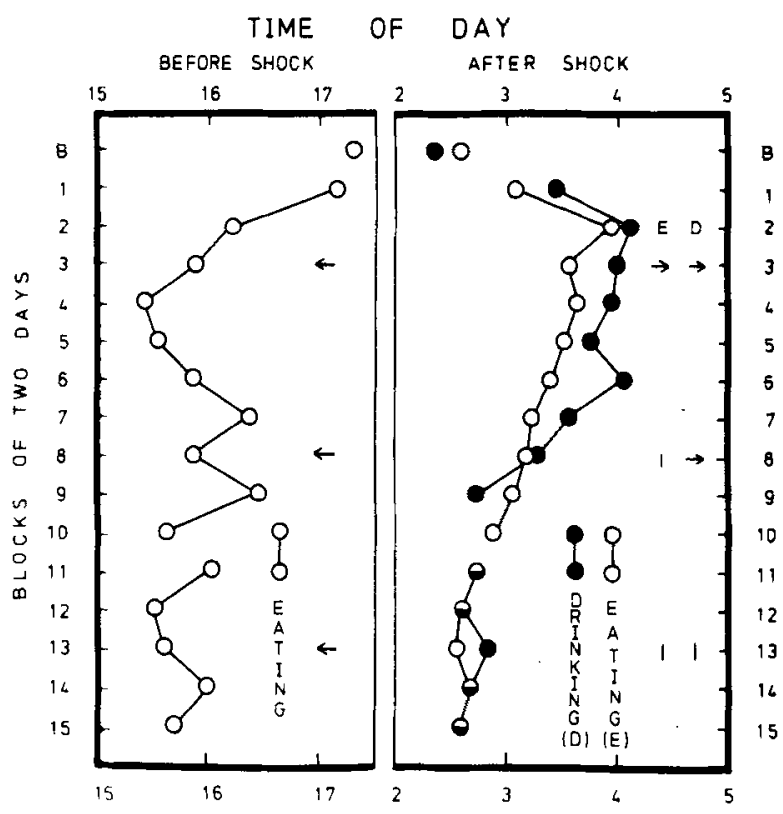

Figure 6. Changes in the means of the midpoint values of eating and drinking in blocks of 2 days for the period of $2113-0800 \mathrm{~h}$ (right) and of eating for the period of 1400-2000 h (eft). Mean midvalue for drinking was not calculated for Block 10 because of a high percentage (41.6\%) of missing data. Block B (base) is a 4-day block. earlier daytime. This finding may best be explained by the circadian timing system (Terman et al., 1984) described above. Midpoint values for drinking during this period were not calculated, because so little drinking took place during this peirod. The brief removal of the subject from the box during the period of 1345-1400 $\mathrm{h}$ may have affected the pattern shown in the left panel of Figure 6. However, similar displacement was observed for eating in the previous single-rat experiment (Imada et al., 1983), in which the rat was never taken out of the apparatus at any time throughout the 10-day shock phase. (4) The results for the first 10 days of the shock phase were the same as those obtained for one rat in the previous experiment for which a more detailed frequency distribution of eating and drinking for $24 \mathrm{~h}$ was found (see Figure 3, Imada et al., 1983). (5) Although the aftereffects of shocks seem to have dissipated by the end of the 20-day shock phase, the anticipatory effect that appeared during the light phase was long lasting and was carried over into the 10-day nonshock phase.

The fact that the 2100 -h shocks had wide-ranging effects on consummatory behavior and general activity, both before and after the shock delivery (Figures 5 and 6), is in sharp contast to Ghiselli and Patton's (1976) findings. They found that 2-h Sidman avoidance training had virtually no effect upon the general activity of rats during the remaining $22 \mathrm{~h}$ of the day, and that the nocturnal rhythm remained unaffected. It should, however, be noted that the avoidance training in Ghiselli and Patton's study took place off-baseline or in a chamber different from the one in which activity was monitored.

One of the major concerns of the present experiment was to observe if rats, during 20-day shock treatment, 
would show any sign of making up the weight loss caused by the suppression of eating and drinking due to shocks given at a favored mealtime. Did they develop any new strategy to enhance the probability of survival? The answer seems to be partly affirmative and partly negative. The facts on the affirmative side are: (1) There were tendencies for body weight to recover slightly (top panel of Figure 2), for the amount of consummatory behavior to increase slightly (lower panels of Figure 2), and for the pattern of consummatory behaviors to return to the normal pattern (right half of Figure 6) during the latter half of the 20-day shock phase. (2) Rats seem to have developed some internal mechanism for efficient nutrient utilization. (3) Compensatory drinking was observed during the light phase (Figure 2). On the negative side, despite the presence of the safe 12-h light phase, total amount of food intake remained suppressed, and hence body weight did not recover completely during the 20-day shock phase. During the light phase, compensatory eating did not occur as it did with drinking (Figure 2).

Finally, it is of interest to note the development of two anticipatory effects which extinguished very little throughout the experiment, suggesting the associative nature of the effects. The first involved the suppression of consummatory behaviors during the period of $2000-2100 \mathrm{~h}$ (Figure 3 ) and the acceleration of general activity for the period of 2000-2012 h (Figure 4). The second was the suppression of eating toward the end of the light phase (Figure 6).

Taking the present results altogether, it was found that administering the 2100 -h shocks within the context of the continuous flow of daily activity and ingestion patterns led to behavioral changes both proximal to and remote from shock presentation, as well as to changes in overall daily ingestion levels that strongly affected long-term weight regulation. Some effects were associative in nature. Some findings were not consisent with those that had usually been observed in ordinary short-session experiments. The nocturnal rhythm, however, was essentially unaffected. Conditions under which rats cannot survive unless they abandon their nocturnality, and their coping attempts under such conditions, will be investigated in the future.

\section{REFERENCES}

Bolles, R. C., \& Fanselow, M. S. (1980). A perceptual-defensiverecuperative model of fear and pain. The Behavioral and Brain Sciences, 3, 291-323.

Bouton, M. E., \& Bolles, R. C. (1980). Conditioned fear assessed by freezing and by the suppression of three different baselines. Animal Learning \& Behavior, 8, 429-434.
Collier, G. H., Hirsch, E., \& Hamlin, P. (1972). The ecological determinants of reinforcement in the rat. Physiology \& Behavior, 9 , 705-716.

Coller, G. H., \& Rovee-Collier, C. K. (1981). A comparative analysis of optimal foraging behavior: Laboratory simulations. In A. C. Kamil \& T. D. Sargent (Eds.), Foraging behavior: Ecological, ethological, and psychological approaches (pp. 39-76). New York: Garland Press.

Davitz, J. R. (1955). Reinforcement of fear at the beginning and at the end of shock. Journal of Comparative and Physiological Psychology, 48, 152-155.

Fitzsimons, T. J., \& Le Magnen, J. (1969). Eating as a regulatory control of drinking in the rat. Journal of Comparative and Physiological Psychology, 67, 273-283.

Ghiselli, W. B., \& Patton, R. A. (1976). Diurnal variation in performance of free-operant avoidance behavior of rats. Psychological Reports, 38, 83-90.

HuRSH, S. R. (1980). Economic concepts for the analysis of behavior. Journal of the Experimental Analysis of Behavior, 34, 219-238.

IMADA, H., \& NAGEISHI, Y. (1982). The concept of uncertainty in animal experiments using aversive stimulation. Psychological Bulletin, 91 , 573-588.

IMADA, H., \& SoGA, M. (1971). The CER and BEL as a function of predictability and escapability of an electric shock. Japanese Psychological Research, 13, 115-122.

Imada, S., Fumi, M., Nakagawa, R., Iso, H., Sugioka, K., \& IMADA, H. (1983). An attempt to measure effects of electric shock upon rat's drinking, eating and general activities over 24 hours a day. Japanese Psychological Research, 25, 52-57.

JACOB, W. J., \& LOLORDO, V. M. (1977). The sensory basis of avoidance responding in the rat: Relative dominance of auditory or visual warning signals and safety signals. Learning and Motivation, 8, 448-466.

JACOB, W. J., \& LoLoRDO, V. M. (1980). Constraints on Pavlovian aversive conditioning: Implications for avoidance learning in the rat. Learning and Motivation, 11, 427-455.

Moran, G. (1975). Severe food deprivation: Some thoughts regarding its exclusive use. Psychological Bulletin, 82, 543-557.

Rashotte, M. R., \& O'Connell, J. M., \& Beidler, D. L. (1982). Associative influence on the foraging behavior of pigeons (Columba livia). Journal of Experimental Psychology: Animal Behavior Processes, 8, 142-153.

Seligman, M. E. P. (1968). Chronic fear produced by unpredictable electric shock. Journal of Comparative and Physiological Psychology, 66, 402-411.

Seligman, M. E. P., Maier, S. F., \& Solomon, R. L. (1971) Unpredictable and uncontrollable aversive events. In F. R. Brush (Ed.), Aversive conditioning and learning (pp. 347-400), New York: Academic Press.

Shishimi, A., \& IMADa, H. (1977). Discriminated and nondiscriminated avoidance conditioning of the rearing response in rats. Animal Learning \& Behavior, 5, 259-264.

Terman, M., Gibbon, J., Fairhurst, S., \& Waring, A. (1984). Daily meal anticipation: Interaction of circadian and interval timing. Annals of New York Academy of Sciences, 423, 470-487.

(Manuscript received August 16, 1984; revision accepted for publication March 1, 1985.) 